
organisationalen Bedürfnisfeld

\author{
Organisationen lernen durch institutionell \\ gestützte Interaktionen voneinander. Durch \\ die Analyse wichtiger Mechanismen für diese \\ Lerneffekte können Empfehlungen für die \\ Institutionalisierung von Nachhaltigkeit \\ formuliert werden. Von Thomas Beschorner \\ und Torsten Behrens
}

$F^{2}$ ür eine nachhaltige Entwicklung besteht es neben den Lernprozessen bei einzelnen Individuen oder innerhalb einzelner Organisationen die Notwendigkeit, gesellschaftliche, das heißt interaktive Lernprozesse zwischen verschiedenen Akteuren anzuregen und durch geeignete Mechanismen zu unterstützen. Für eine solche Betrachtung ist der Institutionenbegriff von zentraler Bedeutung, denn es sind Institutionen, an denen wir unser Handeln orientieren und die für das Gelingen von Interaktionen bedeutsam sind. Institutionen sind nach unserem begrifflichen Verständnis die formalen oder informalen gesellschaftlichen Spielregeln, denen wir gleichwohl nicht nur reaktiv unterworfen sind, sondern die wir durch unser Handeln (re)produzieren. Institutionen sind veränderbar.

Wir haben in einer theoriegeleiteten empirischen Untersuchung auf der Grundlage von Experteninterviews und Literaturstudien die Institutionalisierung von Nachhaltigkeit in den Bedürfnisfeldern Bauen, Wohnen und Mobilität untersucht. Die der Untersuchung zugrunde liegende Grundthese lautet, dass zwischen Organisationen in sogenannten organisationalen (Bedürfnis-)Feldern Angleichungsprozesse, ein Isomorphismus, stattfindet und dass aus der Untersuchung der dafür verantwortlichen Mechanismen wichtige Gestaltungshinweise für gesellschaftliche Lernprozesse abgeleitet werden können.

\section{Organisationale Felder und Institutionalisierung}

Den Theoriehintergrund bildeten insbesondere Elemente aus dem soziologischen Neo-Institutionalismus. Denn mit diesem Ansatz ist es zum einen möglich, Interaktionen zwischen verschiedenen Typen von Organisationen in organisationalen Feldern zu betrachten und den oft unklar verwendeten Begriff des Bedürfnisfeldes als organisationales Bedürfnisfeld analytisch zu schärfen (Beschorner et al. 2005). Dieses versteht sich vereinfacht betrachtet als ein Feld von Organisationen, die gegenseitig aufeinander Bezug nehmen und deren Handlungen auf ein Bedürfnis beziehungsweise eine Basishandlung ausgerichtet sind. Zum anderen ermöglicht es der soziologische Neo-Institutionalismus, die in diesen Feldern wirkenden Mechanismen der Institutionalisierung eingehender zu untersuchen.

\section{Aspekte der Institutionalisierung}

Aus dieser theoretischen Perspektive wurden unter anderem die folgenden Aspekte betrachtet:

- Institutionalisierung durch Isomorphismus: In organisationalen Feldern treten „powerful forces“ auf, die dazu führen, dass Organisationen einander immer ähnlicher werden (DiMaggio 1983/1991). Neben Isomorphismus durch Zwang, zum Beispiel durch Verordnungen und Gesetze, der in der Studie nur nachrangig untersucht wurde, sind dafür mimetischer Isomorphismus und normativer Isomorphismus verantwortlich. Mimetischer Isomorphismus meint, dass sich Organisationen in der Gestaltung ihrer Strukturen und Prozesse an anderen Organisationen orientieren und diese imitieren. Imitationsprozesse können durch organisationsexterne Berater verstärkt werden, die als Diffusionsagenten fungieren. Mit normativem Isomorphismus werden die Folgen zunehmender Professionalisierungen charakterisiert, mit denen eine gemeinschaftliche Denkhaltung und eine berufliche Autonomie von Professionen verbunden sind. Er wird unter anderem durch Ausbildungsinhalte an Hochschulen generiert.

- Institutionalisierung durch Legitimitätsanforderungen: Es wird angenommen, dass Organisationen sich den organisatorischen Umwelten anpassen, indem sie gesellschaftlich legitimierte Elemente in ihre Strukturen integrieren. Hierdurch erlangen sie gesellschaftliche Legitimität und damit jene Ressourcen, die sie für ihr Überleben benötigen (Meyer 1977/1991).

- Institutionalisierung durch institutionelle Entrepreneurs: Der institutionelle Entrepreneur kann die Institutionalisierung von alternativen Prozessen und Strukturen in einem organisationalen Feld forcieren. Er adaptiert nicht die Vorgaben seiner institutionellen Umwelt, sondern gestaltet sie durch nichtregelhaftes Handeln (Galaskiewicz 1991). Institutionelle Entrepreneurs sind von großer Bedeutung für Veränderungsprozesse, denn ihre Aktivitäten wirken nicht nur hinsichtlich konkreter Maßnahmen in einer Organisation, sondern auch auf das gesamte organisationale Feld. 
I Institutionalisierung durch institutionelle Settings: Gesellschaftliche Kontexte können Wahrnehmungen und Interpretationen hervorrufen, die von den Akteuren selbst unabhängig sind und Verhaltensnormen herausbilden, deren Befolgung sich als Orientierungsmodell verselbstständigt. Je stärker ein „social setting“ institutionalisiert ist, desto eher kann mit kultureller Persistenz gerechnet werden, sodass diese Kontexte in der Folge äußerst resistent gegenüber Veränderungen und neuen Institutionalisierungsprozessen sind (Hasse 1999).

\section{Institutionalisierung von Nachhaltigkeit: Isomorphismus und Legitimität}

Mimetischer und normativer Isomorphismus spielen für die Institutionalisierung von Nachhaltigkeit im Bedürfnisfeld Mobilität nur eine geringe Rolle. Im Bereich Bauen und Wohnen, in dem beide Isomorphismen potenziell bedeutsam sein könnten, wird deren Wirkung insbesondere durch eine mangelnde Nachhaltigkeitsorientierung versperrt. Professionals und Berater(innen) stehen zum jetzigen Zeitpunkt einer nachhaltigen Entwicklung eher im Wege, als dass sie diese fördern. Im Handwerk sowie bei Ingenieur(inn)en und Architekt(inn)en wurde eine Grundskepsis gegenüber ökologischen Formen des Bauens konstatiert.

Das Bedürfnisfeld Mobilität ist in einem hohen Maße durch die Inkorporation von Legitimitätsanforderungen der Gesellschaft geprägt. Die Diskussionen um bleifreies Benzin, und Smog haben vor allem seit den 80er Jahren dazu beigetragen, dass der Verkehr als zentraler Verursacher von ökologischen Schäden wahrgenommen wird. Insbesondere Automobilhersteller haben reagiert und Strategien entwickelt, diesen Legitimationsanforderungen zu genügen. Maßnahmen, wie die Einführung des Katalysators oder des Dreiliterautos, aber vor allem die Offenheit gegenüber Nachhaltigkeit auf öffentlicher Bühne, haben es ermöglicht, dass die Automobilität aus der Schusslinie gesellschaftlicher Kontroversen geraten ist - auch wenn faktische ökologische Entlastungen nicht sehr weitreichend stattgefunden haben.

An das Bedürfnisfeld Bauen und Wohnen werden in viel geringerem Maße Legitimitätsanforderungen formuliert, was vermutlich auch auf eine ausgeprägte Regelungsdichte durch Verordnungen und Gesetze zurückzuführen ist, die zu einer geringeren gesellschaftlichen Zuschreibung von Akteursverantwortung führt.

\section{Institutionalisierung von Nachhaltigkeit: Institutionelle Entrepreneurs und Settings}

Institutionellen Entrepreneurs kommt im Bedürfnisfeld Bauen und Wohnen eine untergeordnete Bedeutung zu. Eine Ausnahme bildet hier die Deutsche Energie Agentur, deren Rolle als aktiver Akteur zur Initiierung von Aktionen und Kampagnen im Bereich Klimaschutz herausgestellt wurde. Ein Beispiel sind die Entwicklungen zum standardisierten Energiepass. Gründe für das Fehlen weiterer institutioneller Entrepreneurs sind auch in der klein- und mittelständisch geprägten Struktur des Bedürfnisfeldes zu vermuten. Jene Organisationen weisen ein nur geringes Machtpotenzial zur Beeinflussung des organisationalen Bedürfnisfelds auf.

Im Bedürfnisfeld Mobilität zeigt sich ein anderes Bild, da insbesondere Großunternehmen durch ihre öffentliche Exponiertheit im Bedürfnisfeld Mobilität als potenzielle institutionelle Entrepreneurs in Betracht kommen. Zum jetzigen Zeitpunkt muss jedoch festgestellt werden, dass sie noch nicht als institutionelle Entrepreneurs für eine nachhaltige Entwicklung agieren.

In den untersuchten Bedürfnisfeldern wurden sehr starke institutionelle Settings festgestellt, die einer nachhaltigen Entwicklung im Wege stehen. Im Bedürfnisfeld Mobilität sind Mobilitätsaspekte sehr stark mit Statusaspekten aufgeladen. Freiheit, Flexibilität und Komfort sind weitere Dimensionen, die eine nachhaltige Entwicklung ausbremsen und beispielsweise den Umstieg vom Auto auf öffentliche Verkehrsträger verhindern.

Im Bereich Bauen und Wohnen hemmen die Folgen der demografischen Entwicklung sowie Tendenzen der Suburbanisierung eine nachhaltige Entwicklung. Zudem konnte beobachtet werden, dass der Wunsch nach einem Einfamilienhaus einen ganz wichtigen Wert an sich darstellt. Wir führen den ungebrochenen Wunsch nach einem Eigenheim sowohl auf seine Bedeutung als Statussymbol als auch auf die Tatsache zurück, dass Wohneigentum neuerdings wieder eine zentrale Bedeutung als Element der Altersvorsorge erhalten hat.

\section{Einige Gestaltungsempfehlungen}

Isomorphismus: Im Bereich Bauen und Wohnen könnte das Potenzial genutzt werden, das sich durch die Bedeutung von Professionals und Beratern ergibt. Eine Möglichkeit besteht darin, die Inhalte der Ausbildung von Handwerker(inn)en, Ingenieur(inn)en und Architekt(inn)en hinsichtlich ökologischer Aspekte zu verändern. Eine weitere Möglichkeit wäre die Prämierung ökologischen Bauens durch die Honorarordnung für Architekten und Ingenieure (HOAI). Diese könnte Anreize geben, dem Kunden konkrete Vorschläge für ein ökologisches Bauen und Wohnen zu machen.

Legitimität: Der Bereich Mobilität ist stark von Legitimitätsanforderungen geprägt. Allerdings richten sich die Aktivitäten der Unternehmen bisher eher auf rhetorische und technische Ausweichstrategien, als dass der Kern des Problems angegangen wird. Die Bedeutung der Legitimität könnte besser genutzt werden, wenn gesellschaftliche und politische Organisationen von Unternehmen ein vor allem in Bezug auf Klimaschutz konsequenteres Verhalten einfordern.

Institutionelle Entrepreneurs: Im Bereich Bauen und Wohnen könnten Kooperationen nachhaltigkeitsorientierten Unternehmen und Organisationen zu einem ausreichenden Macht- 
potenzial verhelfen, um Veränderungen auf den Weg zu bringen. Dies ist für den Bereich Mobilität, vor allem für kleinere und mittlere Verbände, ebenfalls eine Option. Große Unternehmen oder zum Beispiel der ADAC besitzen bereits ein großes Machtpotenzial. Bei ihnen müssten motivationale und organisationale Bedingungen für Veränderungen geschaffen werden.

Institutionelle Settings: Die institutionellen Settings spiegeln die kulturellen Werte einer Gesellschaft wider. Auf dieser Ebene Veränderungen vollziehen zu wollen setzt an den eben erwähnten Maßnahmen an, bezieht aber natürlich viele weitere mit ein. Hier sind im Prinzip alle Akteure gefragt, die einen Einfluss auf Lebensstile oder in diesem Falle Mobilitäts- und Wohnstile haben: von den Unternehmen über die Politik, die Medien bis zu den einzelnen Konsument(inn)en.

\section{Schlussbetrachtung}

In der Studie konnte ein Überblick über die Institutionalisierung von Nachhaltigkeit in den jeweiligen Bedürfnisfeldern erarbeitet werden. Es bedarf auch zukünftig großer Anstrengungen, um das Thema Nachhaltigkeit stärker auf die gesellschaftliche Agenda zu bringen. Dies erfordert aus unserer Sicht in besonderem Maße das Zusammenspiel verschiedener Akteure aus Wirtschaft, Politik und Gesellschaft.

Die Studie ist im vergangenen Jahr unter dem Titel „Institutionalisierung von Nachhaltigkeit" im Metropolis-Verlag erschienen.

\section{Literatur}

Beschorner, T. /Behrens, T. / Hoffmann, E. / Lindenthal, A. / Hage, M. / Thierfelder, B. / Siebenhüner, B.: Institutionalisierung von Nachhaltigkeit. Eine vergleichende Untersuchung der organisationalen Bedürfnisfelder Bauen \& Wohnen, Mobilität und Information \& Kommunikation. Marburg 2005.

DiMaggio, P. / Powell, W. W.: The Iron Cage Revisited: Institutional Isomor- phism and Collective Rationality in Organizational Fields, In: DiMaggio, P. (Hrsg.): The New Institutionalism in Organizational Analysis. Chicago, London 1983/1991. S.63-82.

Galaskiewicz, J.: Making Corporate Actors Accountable: Institutional-Building in Minneapolis-St. Paul. In: DiMaggio, P. (Hrsg.): The New Institutionalism in Organizational Analysis. Chicago, London 1991. S.293-310.

Hasse, R. / Krücken, G.: Neo-Institutionalismus. Bielefeld 1999.

Meyer, J. W. / Rowan, B.: Institutionalized Organizations: Formal Structure as Myth and Ceremony. In: DiMaggio, P. (Hrsg.): The New Institutionalism in Organizational Analysis. Chicago, London 1977/1991. S.41-62.

I AUTOREN + KONTAKT

Dr. Thomas Beschorner ist Leiter der wissenschaftlichen Nachwuchsgruppe GELENA an der Carl-von-Ossietzky Universität Oldenburg und Visiting Fellow am Institut für ökologische Wirtschaftsforschung (IÖW), Berlin.

Universität Oldenburg, Fakultät II, Forschungsgruppe GELENA, 26111 Oldenburg. Tel.: 0441/798-438471,

E-Mil: thomas.beschorner@uni-oldenburg.de

Dipl. Oec. Torsten Behrens ist Mitarbeiter der wissenschaftlichen Nachwuchsgruppe GELENA an der Carl-von-Ossietzky Universität Oldenburg.

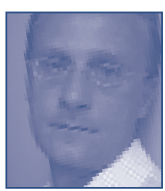

Universität Oldenburg, Fakultät II, Forschungsgruppe GELENA, 26111 Oldenburg. Tel.: 0441/798-4377, E-Mail: torsten.behrens@uni-oldenburg.de

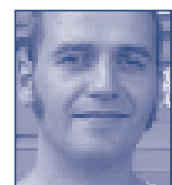

\section{Sustainability}
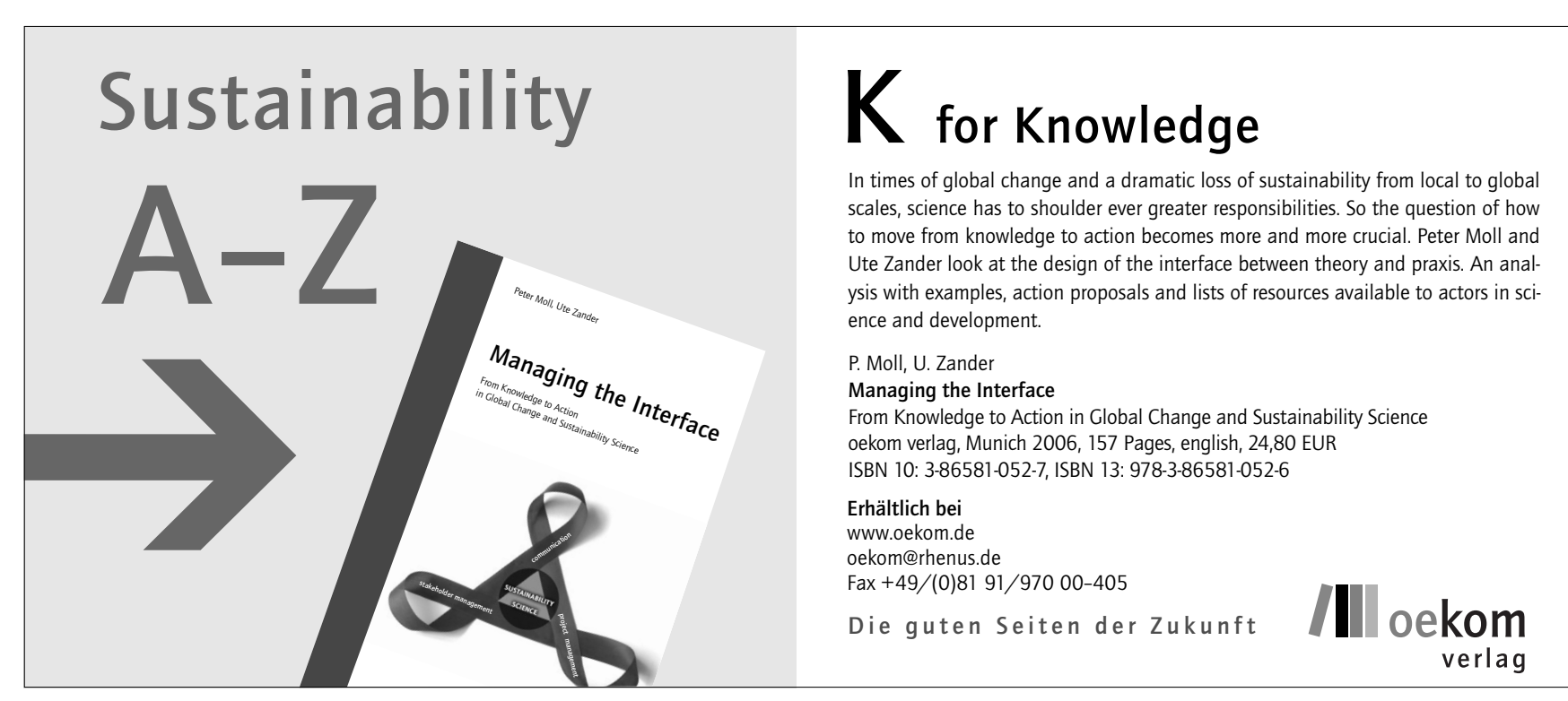

\section{$\mathrm{K}$ for Knowledge}

In times of global change and a dramatic loss of sustainability from local to global scales, science has to shoulder ever greater responsibilities. So the question of how to move from knowledge to action becomes more and more crucial. Peter Moll and Ute Zander look at the design of the interface between theory and praxis. An analysis with examples, action proposals and lists of resources available to actors in science and development.

P. Moll, U. Zander

Managing the Interface

From Knowledge to Action in Global Change and Sustainability Science

oekom verlag, Munich 2006, 157 Pages, english, 24,80 EUR

ISBN 10: 3-86581-052-7, ISBN 13: 978-3-86581-052-6

\section{Erhältlich bei}

www.oekom.de

oekom@rhenus.de

Fax +49/(0)81 91/970 00-405

Die guten Seiten der Zukunft

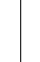

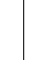

\section{.}
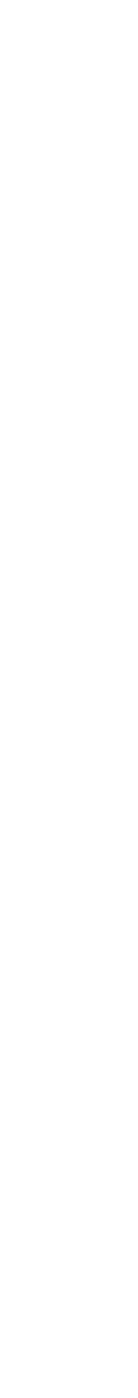

.

.


(c) 20I0 Authors; licensee IÖW and oekom verlag. This is an article distributed under the terms of the Creative Commons Attribution Non-Commercial No Derivates License (http://creativecommons.org/licenses/by-nc-nd/3.o/), which permits unrestricted use, distribution, and reproduction in any medium, provided the original work is properly cited. 